\title{
ANGULAR DIAMETERS OF COOL STARS MEASURED BY THE METHOD OF LUNAR OCCULTATIONS
}

\author{
Nathaniel M. White \\ Lowell Observatory
}

Given the angular diameter and total energy flux of a star, its effective temperature can be determined. Until recently, there were few well-determined angular diameters of cool stars, and effective temperature calibrations rested on angular diameter measurements of several not necessarily "normal" stars. The number of measured angular diameters of stars cooler than about spectral type $G$ is steadily being increased by using the method of photoelectric observations of lunar occultations.

Angular diameter measurements of 42 stars are compiled in the Table. These are the results of many authors; most have been published since 1970. Excluding several geometric occultations (i.e., no diffraction pattern), the mean angular diameter measured has been about 5 milliseconds of arc, with an average error of about $0.8 \mathrm{milliseconds}$ of arc. (Methods of error estimates vary among authors.) This is an indication of the unique power of the lunar occultation technique.

Thirteen of the 42 stars have more than one independent observation, with $\mu$ Gem having eight observations. Since the observational data are recorded in a fraction of a second, are not repeatable, and therefore are unique for a given site and time, duplication of observations at various sites is extremely important and is obviously possible.

As the lack of known cool star angular diameters is being alleviated systematically through the lunar occultation technique, the lack of homogeneous photometry is becoming more important. Photometry reducible to absolute fluxes and covering a wide wavelength range for the stars listed in the Table will provide the 
STELLAR ANGULAR DIAMETERS FROM LUNAR OCCULTATIONS

\begin{tabular}{|c|c|c|c|c|c|c|c|}
\hline BS & Name & $\begin{array}{l}\text { Spectral } \\
\text { Typel }\end{array}$ & $m_{v}{ }^{1}$ & $\begin{array}{l}\text { Millisecon } \\
\quad \phi \cup D\end{array}$ & $\begin{array}{r}\text { ids of } A c^{2} \\
\phi L D\end{array}$ & $\begin{array}{l}\text { Central } \\
\text { Wavelength }\end{array}$ & $\begin{array}{l}\text { Ref. } \\
\text { Code }\end{array}$ \\
\hline $\begin{array}{l}224 \\
601 \\
867\end{array}$ & $\begin{array}{l}63 \delta \text { Psc } \\
45 \mathrm{Ari}\end{array}$ & $\begin{array}{l}\text { K5 II I } \\
\text { gM2 } \\
\text { M6- II I }\end{array}$ & $\begin{array}{l}4^{m} .44 \\
6.11 \\
5.94\end{array}$ & $\begin{aligned} 9.88 & \pm 0.2 \\
11.16 & \pm 0.99\end{aligned}$ & $\begin{array}{c}2.6 \pm 0.5 \\
9.5 \pm 2.0 \\
10.27 \pm 0.21 \\
11.38 \pm 1.01\end{array}$ & $\begin{array}{l}\simeq .5 \\
\simeq .5 \\
\simeq .75 \\
1.64 \\
3.45\end{array}$ & $\begin{array}{r}7 \\
2 \\
1 \\
24 \\
24\end{array}$ \\
\hline 1977 & $\begin{array}{l}+24 \circ 571 \\
\text { Y Tau }\end{array}$ & $\begin{array}{l}\mathrm{K} 5 \\
\mathrm{C} 6,4\end{array}$ & $\begin{array}{l}6.77 \\
\operatorname{Var}\end{array}$ & $\begin{array}{l}1.8 \\
8.39 \pm 0.24 \\
7.98 \pm 0.23\end{array}$ & $\begin{array}{l}8.80 \pm 0.25 \\
8.38 \pm 0.24\end{array}$ & $\begin{array}{l}1.64 \\
1.64\end{array}$ & $\begin{array}{l}24 \\
24\end{array}$ \\
\hline 013 & $\begin{array}{l}B D+27^{\circ} 888 \\
x^{1} \text { Ori }\end{array}$ & $\begin{array}{l}g G 7 \\
G 0 V\end{array}$ & $\begin{array}{l}5.52 \\
4.41\end{array}$ & $2.4 \pm 1.2$ & $1.6 \pm 0.5$ & $\begin{array}{l}\simeq .5 \\
\simeq .70\end{array}$ & $\begin{array}{r}7 \\
12\end{array}$ \\
\hline & $\hat{U}$ Ori & M7. 0-M9.0 & Var & $\begin{array}{l}14.30 \pm 0.54 \\
15.45 \pm 0.33\end{array}$ & $15.45 \pm 0.33$ & $\begin{array}{l}1.64 \\
2.10\end{array}$ & $\begin{array}{l}24 \\
24\end{array}$ \\
\hline 2286 & $\mu \mathrm{Gem}$ & M3 III & 2.97 & $\begin{array}{l}11.8 \pm 2.4 \\
12.3 \pm 1.4 \\
12.1 \pm 1.3 \\
12.0 \pm 0.7 \\
11.8 \pm 0.9 \\
12.3 \pm 1.8 \\
12.3 \pm 0.34 \\
14.2 \pm 1.3\end{array}$ & $\begin{array}{l}13.2 \pm 2.4 \\
13.5 \pm 1.4\end{array}$ & $\begin{array}{c}.456 \\
.820 \\
V \\
V \\
y \\
V \\
.45, .52, .58 \\
V\end{array}$ & $\begin{array}{l}10 \\
10 \\
27 \\
27 \\
27 \\
27 \\
23 \\
20\end{array}$ \\
\hline 2473 & $\varepsilon$ Gem & G8 Ib & 3.08 & $\begin{array}{l}5.6 \pm 0.6 \\
1.8\end{array}$ & & $\simeq .5$ & \\
\hline $\begin{array}{l}2533 \\
2671 \\
2938\end{array}$ & $\begin{array}{l}87 \text { B Gem } \\
\text { R Gem } \\
74 \text { Gem }\end{array}$ & $\begin{array}{l}\text { gK5 } \\
\text { S3,9e-S6,9e } \\
\text { MO III }\end{array}$ & $\begin{array}{l}5.62 \\
6.5 \\
5.07\end{array}$ & $\begin{array}{l}2.8 \\
3.7 \\
2.78 \pm 0.27\end{array}$ & & .75 & $\begin{array}{r}5 \\
28\end{array}$ \\
\hline 3094 & $\begin{array}{l}1 \mathrm{Cnc} \\
\text { V Cnc } \\
+13^{\circ} 1994\end{array}$ & $\begin{array}{l}\text { gK3 } \\
\text { S2.9e } \\
\text { M4 III }\end{array}$ & $\begin{array}{l}5.81 \\
7.5- \\
6.61\end{array}$ & $\begin{array}{l}2.1 \pm 0.6 \\
2.6 \pm 0.7 \\
3.84 \pm 0.55 \\
4.0 \pm 0.5\end{array}$ & $2.8 \pm 0.8$ & $\begin{array}{l}.6 \\
.845 \\
1.64 \\
.75\end{array}$ & $\begin{array}{r}7 \\
16 \\
24 \\
28\end{array}$ \\
\hline $\begin{array}{l}3541 \\
3550 \\
3882\end{array}$ & $\begin{array}{l}x \text { Cnc } \\
60 \text { Cnc } \\
R \text { Leo }\end{array}$ & $\begin{array}{l}\mathrm{C} 5,4 \\
\text { gK5 } \\
\text { gM8e }\end{array}$ & $\begin{array}{l}6.22 \\
5.56 \\
5.0-\end{array}$ & $\begin{array}{l}7.9 \pm 0.8 \\
3.3 \pm 0.4 \\
67 \pm 5\end{array}$ & $9.3 \pm 0.8$ & $\begin{array}{r}.69 \\
.75 \\
\simeq .70\end{array}$ & $\begin{array}{r}4 \\
28 \\
19\end{array}$ \\
\hline $\begin{array}{l}3950 \\
3980\end{array}$ & $\begin{array}{l}\text { II Leo } \\
31 \text { Leo }\end{array}$ & $\begin{array}{ll}\text { M2 } & \text { II I } \\
\text { K4 } & \text { I I I }\end{array}$ & $\begin{array}{l}4.71 \\
4.43\end{array}$ & $\begin{array}{l}5.2 \pm 0.5 \\
2.8 \pm 0.6\end{array}$ & $5.9 \pm 0.5$ & $\begin{aligned} & R \\
\simeq & .55 \\
\simeq & 5\end{aligned}$ & $\begin{array}{r}25 \\
7 \\
73\end{array}$ \\
\hline $\begin{array}{l}4127 \\
4432\end{array}$ & $\begin{array}{l}46 \text { Leo } \\
87 \text { Leo }\end{array}$ & $\mathrm{K} 4_{\mathrm{KM} 2}^{\mathrm{III}}$ & $\begin{array}{l}5.54 \\
5.07\end{array}$ & $\begin{array}{l}3.1 \pm 0.2 \\
5.6 \pm 1.1 \\
3.7 \pm 0.4\end{array}$ & $4.1 \pm 0.4$ & $\begin{array}{l}.51 \\
.547\end{array}$ & $\begin{array}{r}21 \\
9\end{array}$ \\
\hline 4517 & $\nu$ Vir & MI III & 4.02 & & $5.65 \pm 0.05$ & $y$ & $\begin{array}{l}28 \\
22\end{array}$ \\
\hline 4902 & $\psi$ Vir & M3 III & 107 & $4.92 \pm 0.38$ & $\begin{array}{l}6.1 \pm 0.3 \\
6.5 \pm 0.3 \\
5.11 \pm 0.39\end{array}$ & $\begin{array}{r}.63 \\
.69 \\
1.64\end{array}$ & $\begin{array}{l}26 \\
12 \\
24\end{array}$ \\
\hline 5301 & & gM3 & 4.91 & $\begin{array}{l}4.65 \pm 0.33 \\
5.0 \pm 1 .\end{array}$ & $\begin{array}{l}3.6 \pm 0.5 \\
4.79 \pm 0.34\end{array}$ & $\begin{array}{c}\simeq .80 \\
1.64 \\
\mathrm{~b}\end{array}$ & $\begin{array}{r}2 \\
24 \\
28\end{array}$ \\
\hline $\begin{array}{l}5824 \\
6134\end{array}$ & $\begin{array}{l}42 \mathrm{Lib} \\
\alpha \mathrm{Sco} \\
\text { IRC-20 } 321 \\
\text { V774 Sgr }\end{array}$ & $\begin{array}{ll}\text { K4 } & \text { I I I } \\
\text { M7 } & \text { Ib } \\
\text { M6 } & \\
\text { M5 } & \end{array}$ & $\begin{array}{r}4.95 \\
1.08 \\
12 .-\end{array}$ & $\begin{array}{l}2.2 \pm 0.3 \\
41 . \pm 1 . \\
4.37 \pm 0.25 \\
5.65 \pm 0.73\end{array}$ & & $\begin{array}{l}2.2 \\
1.64\end{array}$ & $\begin{array}{l}15 \\
11 \\
24 \\
24\end{array}$ \\
\hline
\end{tabular}




\section{STELLAR ANGULAR DIAMETERS FROM LUNAR OCCULTATIONS (continued)}

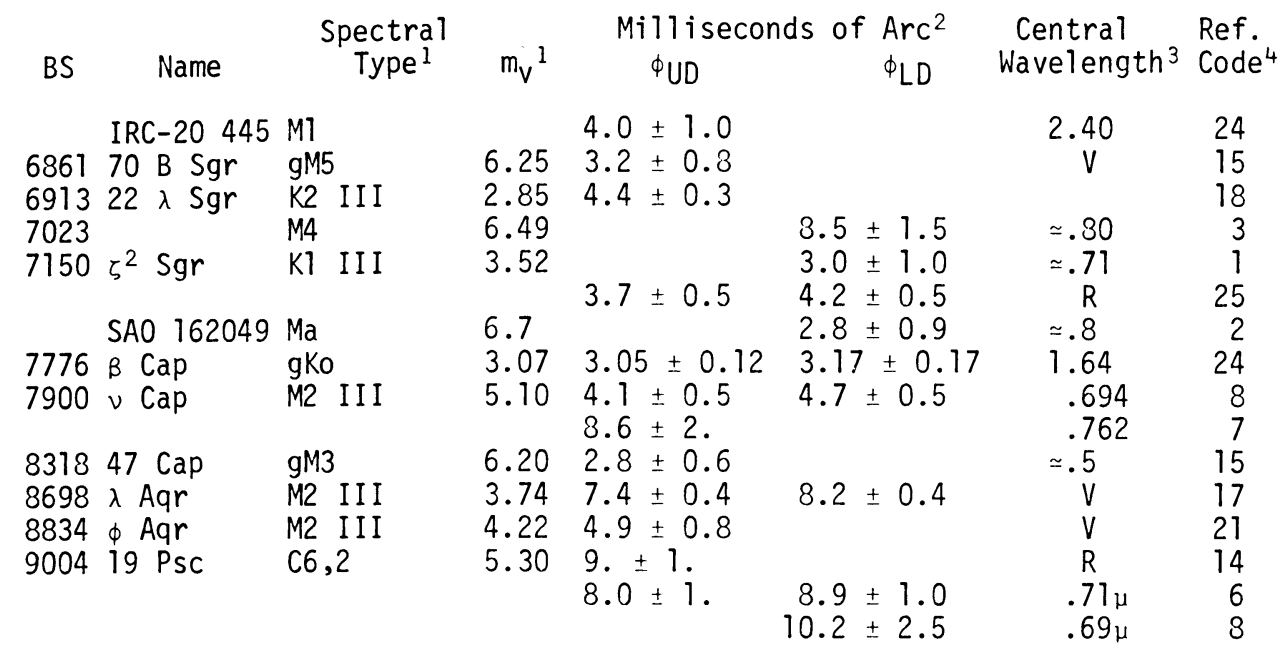

NOTES TO TABLE

${ }^{1}$ Spectral types and magnitudes are from the reference listed or the Bright Star Catalog.

${ }^{2} \phi U D$ and $\phi_{L D}$ indicate the fitted model used, either a uniform disk or limbdarkened disk approximation, respectively. Nearly all limb-darkened fits used the fully darkened assumption, i.e., $\beta=1.0$.

${ }^{3}$ The approximate central wavelengths are in micron units, with the letters being the usual notation for standard photometric systems.

${ }^{4}$ The five angular diameters with reference code 28 , white, N. M. (1978), have not been published previously.

best estimate of their total flux output. Since the effective temperature is a function of the fourth root of the total flux, the error in effective temperature determinations will be reduced to mostly that related to the angular diameter measurement, that is, about $5 \%$ in the effective temperature.

The purpose of this compilation is to draw attention to these stars (some of which do not even have good MK spectral types), in order to encourage their detailed observational and theoretical investigation. 


\section{REFERENCES TO TABLE}

1. Africano, J. L., Cobb, C. L., Dunham, D. W., Evans, D. S., Fekel, F. C., and Vogt, S. S. (1975). Astron. J. 80, 689.

2. Africano, J. L., Evans, D. S., Fekel, F. C., and Ferland, G. J. (1976). Astron. J. 81, 650.

3. Africano, J. L., Evans, D. S., Fekel, F. C., and Montemayor, T. (1977). Astron. J. 82, 631.

4. Bartholdi, P., Evans, D. S., Mitchel1, R. I., Silverberg, E.C., Wells, D. C., and Wiant, J. R. (1972). Astron. J. 77, 256.

5. Beavers, W. I., and Eitter, J. J. (1974). Bul1. Amer. Astron. Soc. $6,216$.

6. de Vegt, Chr. (1974). Astron. Astrophys. 34, 457.

7. de Vegt, Chr. (1976). Astron. Astrophys. 47, 457.

8. Dunham, D. W., Evans, D. S., Silverberg, E. C., and Wiant, J. R. (1975). Astron. J. 78, 199.

9. Dunham, D. W., Evans, D. S., and Sandmann, W. H. (1974). Astron. J. 79, 483.

10. Dunham, D.W., Evans, D.S., and Vogt, S.S. (1975). Astron. J. $80,45$.

11. Evans, D. S. (1957). Astron. J. 62, 83.

12. Evans, D. S., Fekel, F. C., and Silverberg, E. C. (1977). Astron. J. 82, 828.

13. GTass, I. S., and Morrison, L. V. (1976). Monthiy Notices Roy. Astron. Soc. 175, 57.

14. Lasker, B. M., Bracker, S. B., and Kunkel, W. E. (1973). Pub1. Astron. Soc. Pacific 85, 109.

15. Harwood, J.M., Nather, R.E., Walker, A.R., Warner, B., and Wild, P.A.T. (1975). Monthly Notices Roy. Astron. Soc. 170, 229.

16. McGraw, J. T., and Angel, J.R.P. (1974). Astron. J. 79, 485.

17. Nather, R. E., McCants, M. M., and Evans, D. S. (1970). Astrophys. J. 160, L181.

18. Nather, R. E. (1972). Ph.D. dissertation, Univ. of Cape Town.

19. Nather, R. E., and Wild, P.A.T. (1973). Astron. J. 78, 628.

20. Nelson, M. R. (1975). Astrophys. J. 198, 127.

21. Poss, H. L. (1971). in Highlights of Astronomy, Vol. 2, C. de Jager, ed., D. Reidel, Dordrecht, P. 692.

22. Rakos, K. D. (1971). in Highlights of Astronomy, Vol. 2, C. de Jager, ed., D. ReideT, Dordrecht, p. 675.

23. Ridgway, S. T., Wells, D. C., and Carbon, D. F. (1974). Astron. J. 79, 1079.

24. Ridgway, S. T., Wells, D. C., and Joyce, R. R. (1977). Astron. J. 82, 414.

25. Vilas, F., and Lasker, B. M. (1977). Publ. Astron. Soc. Pacific $89,95$.

26. Walker, A.R. (1975). Monthly Notices Roy. Astron. Soc. 173, $29 \mathrm{P}$.

27. White, N. M. (1974). Astron. J. 79, 1076.

28. White, N. M. (1978). In preparation. 